\title{
Type-II Intermittency in a Class of Two Coupled One-Dimensional Maps
}

\author{
J. LAUGESEN ${ }^{\mathrm{a}}$, E. MOSEKILDE ${ }^{\mathrm{a}, *}$, T. BOUNTIS ${ }^{\mathrm{b}}$ and S. P. KUZNETSOV ${ }^{\mathrm{c}}$ \\ ${ }^{a}$ Department of Physics, The Technical University of Denmark, 2800 Lyngby, Denmark; ${ }^{b}$ Department of Mathematics, \\ University of Patras, Patras 26110, Greece; ${ }^{\mathrm{c} I n s t i t u t e}$ of Radio-Engineering and Electronics, \\ Russian Academy of Sciences, Zelenaya 38, Saratov 410019, Russia
}

(Received 15 March 2000)

\begin{abstract}
The paper shows how intermittency behavior of type-II can arise from the coupling of two one-dimensional maps, each exhibiting type-III intermittency. This change in dynamics occurs through the replacement of a subcritical period-doubling bifurcation in the individual map by a subcritical Hopf-bifurcation in the coupled system. A variety of different parameter combinations are considered, and the statistics for the distribution of laminar phases is worked out. The results comply well with theoretical predictions. Provided that the reinjection process is reasonably uniform in two dimensions, the transition to type-II intermittency leads directly to higher order chaos. Hence, this transition represents a universal route to hyperchaos.
\end{abstract}

Keywords: Type-II intermittency; Two-dimensional maps; Hyperchaos; Absorbing area

\section{INTRODUCTION}

Many interesting phenomena have been discovered by studying the dynamics of weakly coupled, identical one-dimensional (1-D) maps. In particular, coupled logistic [1-3] and coupled circle maps [4] have been extensively investigated from the point of view of the persistence of their bifurcation structures under the stabilizing or destabilizing influences of the mutual interaction.

It was observed by Frøyland [1], for instance, that the period-doubling route to chaos may be replaced by a quasiperiodic transition when two identical logistic maps are coupled symmetrically. The reason for this is that at an early stage of the period-doubling cascade, a Hopf-bifurcation on the antisymmetric solution appears instead of period doubling. A similar replacement was found experimentally by Van Buskirk and Jeffries [5] who studied driven electronic resonance circuits consisting of an inductance in series with a pnjunction acting as a nonlinear capacitance. When varying the drive voltage the single resonator showed a period-doubling transition to chaos,

*Corresponding author. 
whereas resistively coupled resonance circuits exhibited a quasiperiodic transition. A qualitative explanation of this phenomenon was provided by Erastova and Kuznetsov [6]. Based on detailed analytic work, Reick and Mosekilde [7] were subsequently able to demonstrate that the behavior is generic to symmetrically coupled perioddoubling systems, and that it is robust towards slight deviations from complete symmetry.

In parallel studies, Fujisaka and Yamada [8] have shown how two identical chaotic oscillators under variation of the coupling strength can attain a state of chaotic synchronization, and a variety of applications of this phenomenon for chaos suppression, for monitoring of dynamical systems, and for different communication purposes have been suggested $[9,10]$. Most recently, the formation of riddled basins of attraction [11-13] and the associated phenomenon of on-off intermittency in systems of two coupled 1-D maps $[14,15]$ have attracted significant interest.

By contrast to the various bifurcation scenarios described above, the dynamics of coupled 1-D maps does not appear to have been as thoroughly analyzed in the cases where the individual map follows the intermittency route to chaos. In their classic paper on intermittent transition to turbulence in dissipative systems, Pomeau and Manneville [16] introduced three different types of intermittency, using a classification that reflects the types of local bifurcation by which a periodic orbit can lose its stability. Type-I and type-III intermittencies are associated with the passage of a real Floquet multiplier through +1 and -1 , respectively. These types of intermittency have been theoretically explained in terms of simple 1-D maps [17], and they have also been widely demonstrated in the laboratory $[18,19]$.

Type-II intermittency is essentially a two-dimensional phenomenon. It arises when a periodic orbit loses its stability in a subcritical Hopf-bifurcation, provided that a global reinjection mechanism exists that can send the trajectory back into the neighborhood of the destabilized periodic orbit. This type of intermittency has only been observed in a limited number of experiments [20,21], and it appears that simple, theoretical examples are lacking. Most of the existing models are high dimensional, and the strongly nonuniform reinjection processes that they produce cause the experimental (or numerical) results for the scaling behavior to deviate from simple theoretical calculations [22].

The purpose of the present paper is to show how type-II intermittency can arise in a class of weakly coupled 1-D maps which individually exhibits type-III intermittency. In much the same way as the coupling of two logistic maps can lead to the appearance of a Hopf-bifurcation instead of a period doubling, the coupling of the two maps in the present case leads to the replacement of a subcritical period doubling by a subcritical Hopfbifurcation. We demonstrate that within this class of maps, type-II intermittency is a generic phenomenon, in that it occurs for various types of coupling, for large ranges of parameter values, and for arbitrarily small coupling constants. The statistics for the distribution of laminar phases is worked out, and our numerical results compare favorably with theoretical predictions. Provided that reinjection near the unstable two-dimensional manifold is reasonably uniform, there is a tendency for the transition to type-II intermittency to be associated with the abrupt generation of hyperchaos.

\section{TYPE-II INTERMITTENCY IN COUPLED 1-D MAPS}

Let us start by considering the 1-D map

$$
x_{n+1}=-\left((1+\mu) x_{n}+x_{n}^{3}\right) e^{-b x_{n}^{2}}
$$

with the bifurcation parameters $\mu$ and $b>0$. For $b=0$, the map represents the normal form of a subcritical period-doubling bifurcation with a stable fixed point $x^{*}=0$ surrounded by an unstable period-2 orbit $x_{1,2}= \pm \sqrt{-\mu}$ for $\mu<0$, and with an unstable fixed point for positive values of $\mu$. Combined with the reinjection mechanism provided by the exponential factor with $b>0$, the subcritical period doubling produces the typical behavior of type-III intermittency. 
We have previously studied this phenomenon in detail and found that the strongly nonuniform reinjection process produced by the map (2.1) causes an anomaly in the statistics of the laminar phases [23]. This nonuniformity arises because the singularity associated with the maximum of the map controls the behavior around the minimum reinjection distance. To allow a better regulation of the reinjection process, the map may be modified to read

$$
x_{n+1}=f\left(x_{n}\right)=-\left((1+\mu+\gamma) x_{n}+x_{n}^{3}\right) e^{-b x_{n}^{2}}+\gamma x_{n} .
$$

Around the fixed point, this map has precisely the same behavior as (2.1). However, by varying $\gamma$ one can shift the position of the most probable reinjection point relative to the unstable fixed point and in this way achieve a more homogeneous reinjection process.

Figure 1 shows the form of the map (2.2) for $b=0.14, \mu=0.005$, and $\gamma=0.02$. Indicated on the figure is the critical point $x_{c} \cong-3.167$ together with its first and second iterates, $f\left(x_{c}\right)$ and $f^{2}\left(x_{c}\right)$ respectively. With the assumed parameter values, $f\left(x_{c}\right) \cong 8.534$ falls to the right of the point $f\left(x_{1}\right) \cong 7.546\left(=-x_{0}\right)$ where the map crosses the horizontal axis. Hence, $f^{2}\left(x_{c}\right)>0$, and reinjection of the second iterate of the critical point directly into the unstable fixed point does not occur. Together with a point $x_{2}\left(f\left(x_{2}\right)=f\left(x_{1}\right)\right)$ to the left of $x_{c}, x_{1}$ denotes the point whose second iterate falls in the unstable fixed point. For the reinjection process to be reasonably uniform, the average (numerical) slope of the map in $x_{1}$ and $x_{2}$ (weighted by the respective visiting frequencies) times the slope in $f\left(x_{1}\right)$ should be close to 1 . This condition is approximately satisfied with the above parameters.

Let us hereafter turn our attention towards the following system of two coupled 1-D maps

$$
\begin{aligned}
x_{n+1}= & -\left((1+\mu+\gamma) x_{n}+x_{n}^{3}\right) e^{-b x_{n}^{2}} \\
& +\gamma x_{n}+\varepsilon m\left(x_{n}, y_{n}\right)
\end{aligned}
$$

and

$$
\begin{aligned}
y_{n+1}= & -\left((1+\mu+\gamma) y_{n}+y_{n}^{3}\right) e^{-b y_{n}^{2}}+\gamma y_{n} \\
& +\varepsilon_{n} n\left(x_{n}, y_{n}\right)
\end{aligned}
$$

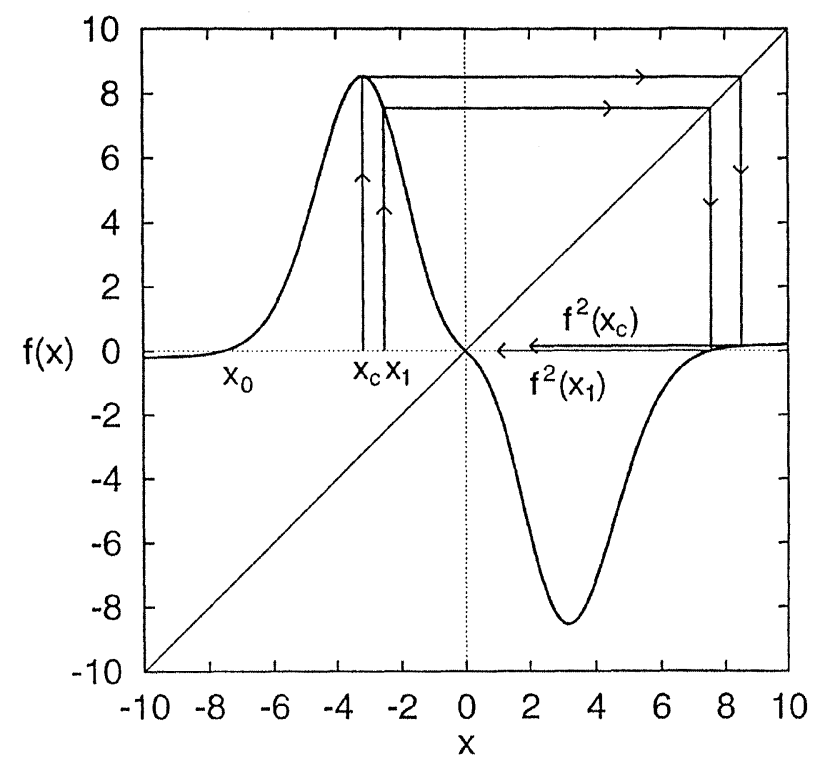

FIGURE 1 Sketch of the $\operatorname{map} f(x)=-\left((1+\mu+\gamma) x_{n}+x_{n}^{3}\right) e^{-b x_{n}^{2}}+\gamma x_{n}$ with $b=0.14, \mu=0.005$ and $\gamma=0.02$. For these parameter values, the reinjection process is approximately uniform in an interval of the order of $f^{2}\left(x_{c}\right) \cong 0.147$ on either side of the unstable fixed point. 
where the functions $m\left(x_{n}, y_{n}\right)$ and $n\left(x_{n}, y_{n}\right)$ specify the form of the coupling, and $\varepsilon$ determines its strength. In order for the coupled system to show type-II intermittency, the linear terms of the coupling functions in the neighborhood of the fixed point must have a form such that the subcritical period doubling in the individual map is replaced by a subcritical Hopf-bifurcation in the coupled system.

To find the condition for this to occur we determine the Jacobian matrix at the fixed point $\left(x_{n}, y_{n}\right)=(0,0)$ and calculate its eigenvalues

$$
\begin{aligned}
& \lambda_{1,2}=-(1+\mu) \\
&+\frac{\varepsilon}{2}\left[\left(\frac{\partial m}{\partial x}+\frac{\partial n}{\partial y}\right)\right. \\
& \quad \pm \sqrt{\left.\left(\frac{\partial m}{\partial x}-\frac{\partial n}{\partial y}\right)^{2}+4 \frac{\partial m}{\partial y} \frac{\partial n}{\partial x}\right] .}
\end{aligned}
$$

For symmetric coupling $((\partial m / \partial x)=(\partial n / \partial y)$ and $(\partial m / \partial y)=(\partial n / \partial x))$ we have

$$
\lambda_{1,2}=-(1+\mu)+\varepsilon\left(\frac{\partial m}{\partial x} \pm \frac{\partial m}{\partial y}\right) .
$$

Under these conditions, the eigenvalues are always real, and a Hopf-bifurcation cannot occur. The criterium for the eigenvalues to become complex is

$$
-4 \frac{\partial m}{\partial y} \frac{\partial n}{\partial x}>\left(\frac{\partial m}{\partial x}-\frac{\partial n}{\partial y}\right)^{2} .
$$

Hence, $(\partial m / \partial y)$ and $(\partial n / \partial x)$ must have opposite signs. For an antisymmetric coupling with $(\partial n /$ $\partial x)=-(\partial m / \partial y)=a$ and $(\partial m / \partial x)=-(\partial n / \partial y)=d$ we have

$$
\lambda_{1,2}=-(1+\mu) \pm i \varepsilon \sqrt{a^{2}-d^{2}},
$$

and the eigenvalues are complex conjugate as long as $a^{2}>d^{2}$.

The bifurcation point, i.e., the value of $\mu$ for which $\left|\lambda_{1,2}\right|=1$ is given by

$$
\mu_{0}=-1+\sqrt{1-\varepsilon^{2}\left(a^{2}-d^{2}\right)} \cong-\frac{1}{2} \varepsilon^{2}\left(a^{2}-d^{2}\right) .
$$

As a specific example in the following numerical study we shall take $m\left(x_{n}, y_{n}\right)=y_{n}$ and $n\left(x_{n}, y_{n}\right)=$ $-x_{n}$ corresponding to $a=-1$ and $d=0$. This gives the 2-D map

$$
\begin{array}{r}
x_{n+1}=-\left((1+\mu+\gamma) x_{n}+x_{n}^{3}\right) e^{-b x_{n}^{2}}+\gamma x_{n}+\varepsilon y_{n} \\
y_{n+1}=-((2.10) \\
\end{array}
$$

with the eigenvalues

$$
\lambda_{1,2}=-(1+\mu) \pm i \varepsilon
$$

and the bifurcation point

$$
\mu_{0}=-1+\sqrt{1-\varepsilon^{2}} \cong-\frac{1}{2} \varepsilon^{2} .
$$

To show that the Hopf-bifurcation is subcritical we introduce the complex notation

$$
z_{n}=x_{n}+i y_{n}=\left|z_{n}\right| e^{i \varphi_{n}},
$$

multiply (2.11) by $i$, and add it to (2.10). When retaining terms up to third order in $x_{n}$ and $y_{n}$ this gives

$$
z_{n+1}=-(1+\mu+i \varepsilon) z_{n}-B\left(\frac{3}{4} z_{n}\left|z_{n}\right|^{2}+\frac{1}{4} \bar{z}_{n}^{3}\right) .
$$

Here,

$$
B=1-b(1+\mu+\gamma)
$$

and the overbar denotes complex conjugate. Since the argument of $z_{n}$ changes monotonously during the iteration $\varphi_{n+1} \cong \varphi_{n}+\Delta \varphi_{n}$ with $\Delta \varphi_{n} \cong$ $\tan ^{-1}\{\varepsilon /(1+\mu)\}+\pi$ for $\left|z_{n}\right| \ll 1$, the asynchronous term $\bar{z}_{n}^{3}$ that appears in (2.15) can be neglected. Hence, the expression for $z_{n+1}$ is seen to represent the normal form for a subcritical Hopf-bifurcation, provided that $B>0$ at the bifurcation point $\mu_{0} \cong-(1 / 2) \varepsilon^{2}$. 
Except for the slight shift in the bifurcation point caused by the coupling, this is precisely the same condition that determines whether the period-doubling bifurcation in the individual map is subcritical or not [23]. Hence, for a subcritical Hopf-bifurcation to occur we must have

$$
b<\frac{1}{1-(1 / 2) \varepsilon^{2}+\gamma} .
$$

In the numerical simulations to be discussed below typical parameter values are $b=0.14$, $\gamma=0.02$, and $\varepsilon=0.001$, and the condition (2.17) will always be satisfied.

\section{NUMERICAL RESULTS}

Figure 2 shows an example of the temporal behavior of the coupled map system over a period corresponding to 100,000 iterations. To illustrate the long laminar phases that occur immediately above the subcritical Hopf-bifurcation we have assumed $\mu=0.0001$. The vertical axis shows the variation of $\operatorname{sign}\left(x_{n}\right) \sqrt{\left|x_{n}\right|}$. The nonlinear scale serves to accentuate the variations during the laminar phases relative to the much larger excursions that take place during the turbulent bursts. The spiralling nature of the iteration process characteristic for type-II intermittency is revealed through the relatively slow oscillations that grow in size until the system bursts into a turbulent phase.

Figure 3 shows a phase plot in which we have followed the behavior of the system through 240 reinjection processes. Only each second iterate is displayed. The figure again illustrates the spiralling character of the iteration process in the neighborhood of the unstable fixed point. At the same time, the figure gives an impression of how the reinjection points are distributed.

Figure 4 provides a much more detailed picture of the distribution of reinjection points in phase space. For the assumed parameter values, this distribution is characterized by a relatively homogeneous region around the unstable fixed point.

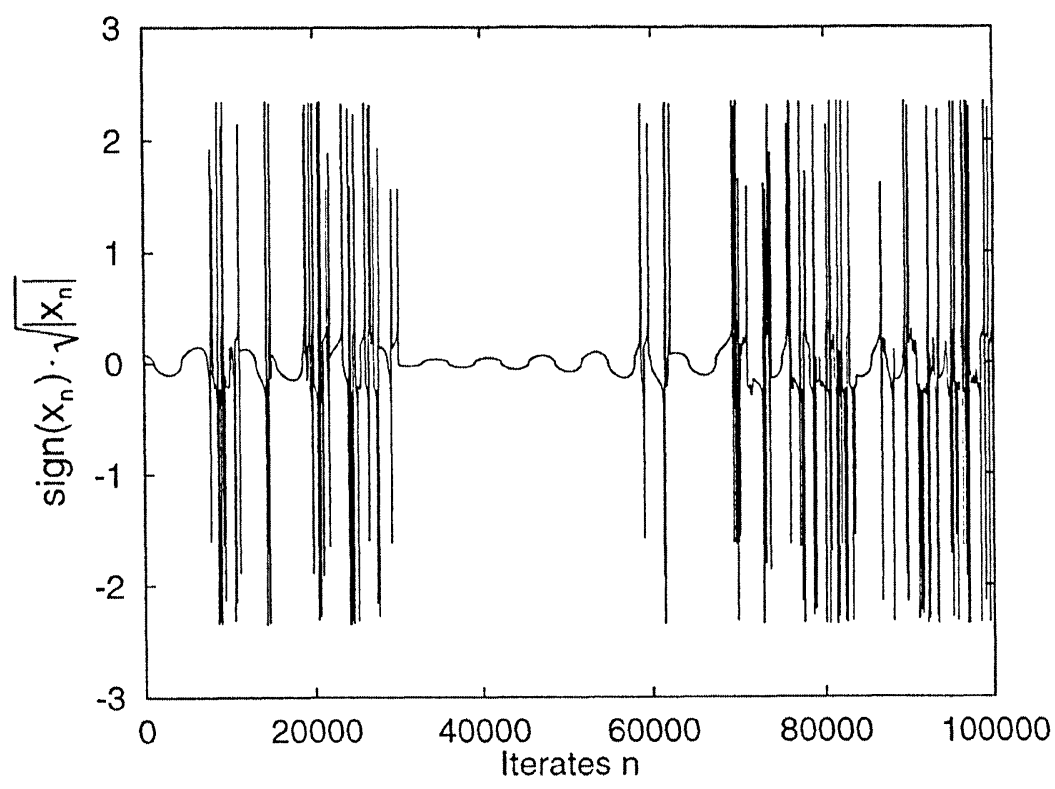

FIGURE 2 Temporal behavior of the coupled map system immediately above the subcritical Hopf-bifurcation. One of the laminar phases is seen to last for nearly 30,000 iterations. Note that the scale on the vertical axis is nonlinear. Only each second iteration is shown. 


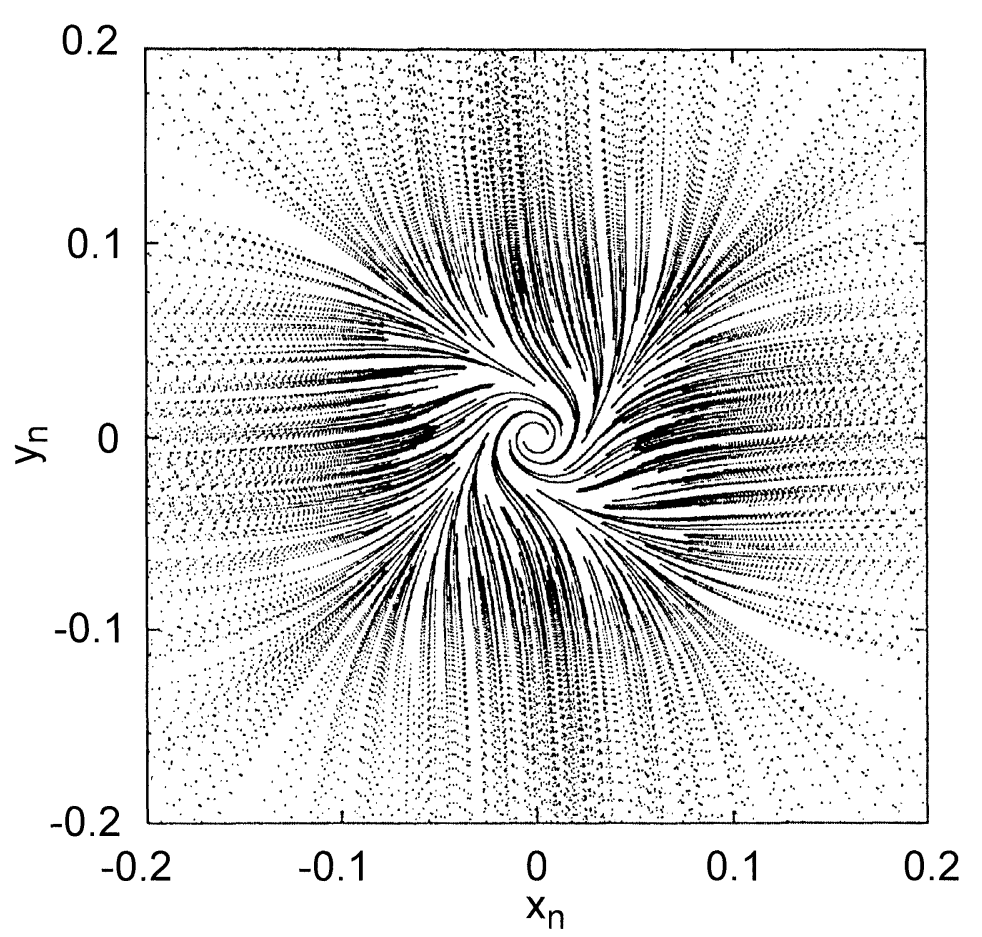

FIGURE 3 Phase plot illustrating the spiralling character of the iteration process for type-II intermittency. The figure was obtained for $\mu=0.0001, b=0.186, \gamma=0.13$, and $\varepsilon=0.001$.

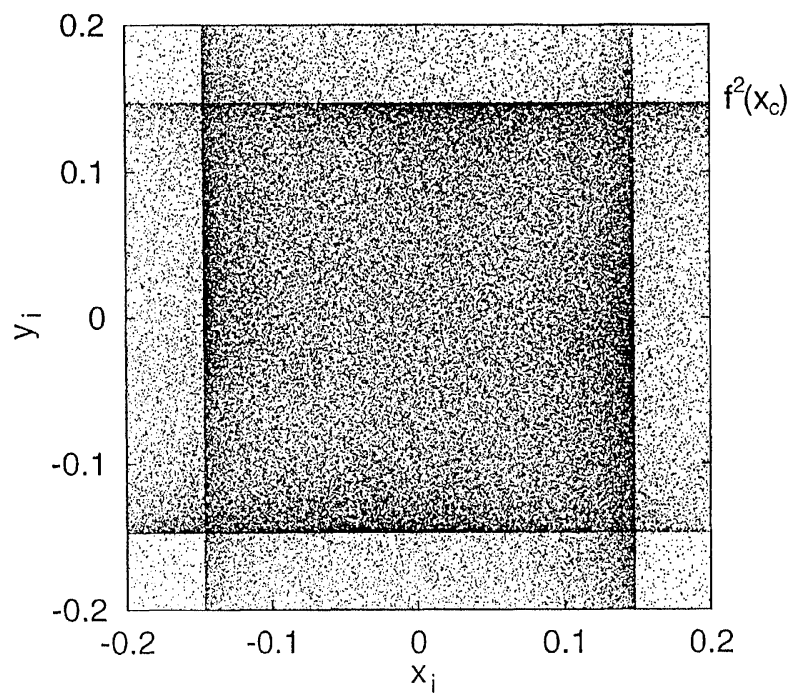

FIGURE 4 Distribution of reinjection points in phase space. Note the region of approximately homogeneous reinjection surrounding the unstable fixed point. Here, $\mu=0.005, \varepsilon=0.001, b=0.14$, and $\gamma=0.02$.

This region arises through the crossing bands extending along the vertical and horizontal axes and bounded by the critical curves of the coupled map system $[24,25]$. In the limit of low coupling, the width of each of these bands is determined by the magnitude of $f^{2}\left(x_{c}\right)$. 
Figures $5 \mathrm{a}$ and $\mathrm{b}$ display the radial and angular distributions of the reinjection points. The radial distribution $P\left(r_{i}\right)=N\left(r_{i}\right) / r_{i}$ is approximately constant up to reinjection distances $r_{i}=\sqrt{x_{i}^{2}+y_{i}^{2}} \cong$ $0.8 f^{2}\left(x_{c}\right)$. For $r_{i} \cong f^{2}\left(x_{c}\right)$ a singularity occurs. This phenomenon is clearly associated with the focussing effect of the map near the critical point. For $r_{i}>f^{2}\left(x_{c}\right)$, the reinjection probability rapidly decreases with a second, although much weaker singularity for $r_{i} \cong \sqrt{2} f^{2}\left(x_{c}\right)$. The angular distribution of the reinjection points (Fig. 5b) was determined for $r_{i}=0.8 f^{2}\left(x_{c}\right)$. The distribution appears at first sight to be approximately uniform, although there is a $10 \%$ modulation with maxima occurring around $\varphi_{i}=p \pi / 2, p=1,2,3$ and 4. However, a closer examination of the angular distribution reveals an extremely complicated structure with singularities associated with the crossing of higher-order critical curves through the reinjection area $[24,25]$. This is
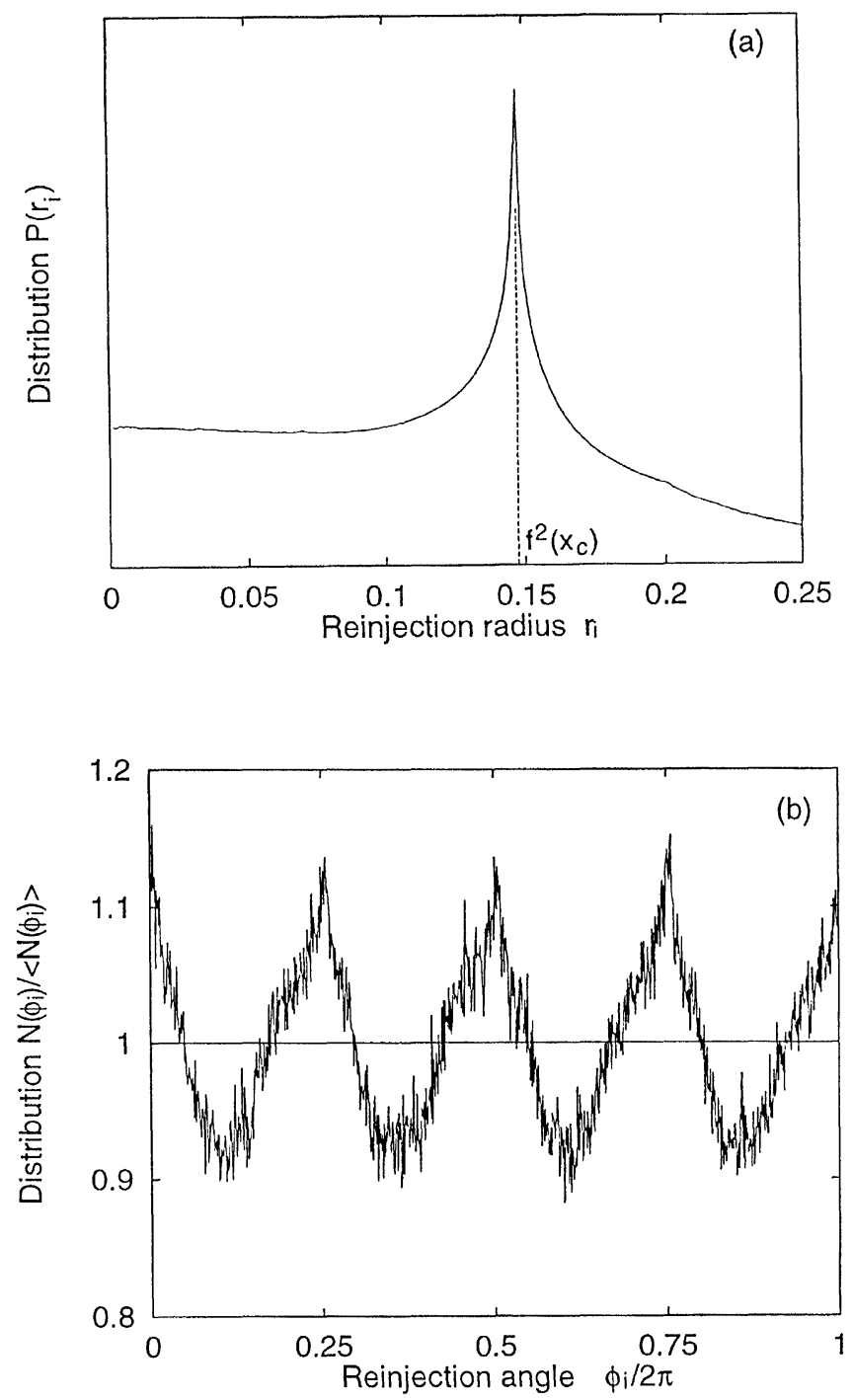

FIGURE 5 Radial (a) and angular (b) distributions of reinjection points. The gross features of the angular distribution is nearly homogeneous (for $r_{i}<0.8 f^{2}\left(x_{c}\right)$ ). The radial distribution displays a couple of singularities for $r_{i} \cong f^{2}\left(x_{c}\right)$ and $r_{i} \cong \sqrt{2} f^{2}\left(x_{c}\right)$ combined with a rapid decline for larger values of $r_{i}$. However, for reinjection distances $r_{i}<0.8 f^{2}\left(x_{c}\right)$, the distribution is nearly homogeneous. Parameters are the same as for Figure 4. 


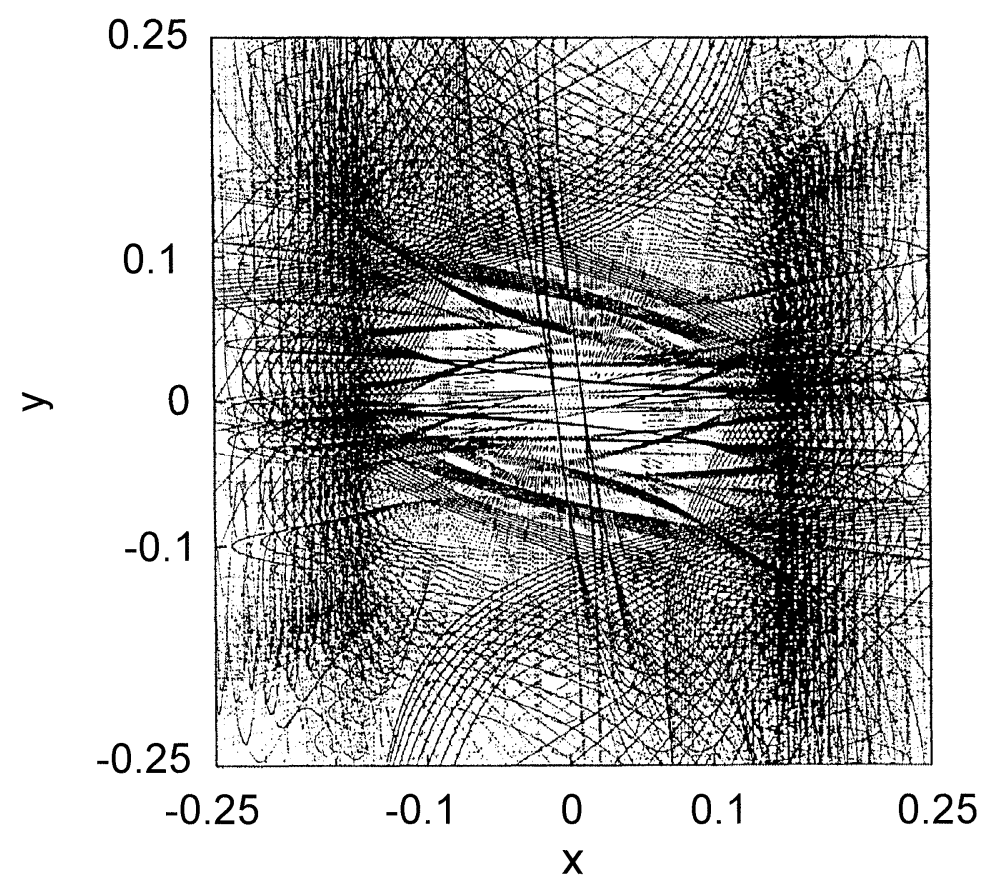

FIGURE 6 Network of critical curves of rank 30-50 crossing the reinjection zone.

illustrated in Figure 6 where we have drawn the network of critical curves of rank $30-50$ in a region around the unstable fixed point. Critical curves of lower rank do not pass this region. Because of variations in the invariant density for the intermittent attractor, the reinjection probability is particularly high in the vicinity of the critical curves. One can also determine the angular variation in the density of critical curves over a distance $r=0.8 f^{2}\left(x_{c}\right)$ from the unstable fixed point. This variation clearly reveals the complicated mechanisms that control the finer aspects of the reinjection process.

\section{DISTRIBUTION OF LAMINAR PHASES}

Close to the fixed point $(0,0)$, the coupled map system $(2.10-2.11)$ may be approximated by

$$
r_{n+1} \cong \alpha r_{n}+\frac{3}{4} B r_{n}^{3}
$$

and

$$
\varphi_{n+1} \approx \varphi_{n}+\tan ^{-1}\{\varepsilon /(1+\mu)\}+\pi
$$

where $\alpha=\sqrt{(1+\mu)^{2}+\varepsilon^{2}} \cong 1+\mu$. As before $r_{n}=\sqrt{x_{n}^{2}+y_{n}^{2}}=\left|z_{n}\right|$, and $B=1-b(1+\mu+\gamma)$. By renormalizing $r_{n}$, (4.1) may be recast into the standard form

$$
r_{n+1}=(1+\mu) r_{n}+r_{n}^{3}
$$

Since the increase in $r_{n}$ per iteration is small, (4.3) may hereafter be replaced by the differential equation

$$
\frac{d r}{d n}=\mu r+r^{3}
$$

from which we calculate the number of iterations $l\left(r_{i}\right)$ in the laminar phase as a function of the reinjection distance [17] 


$$
l\left(r_{i}\right)=-\frac{1}{2 \mu} \ln \frac{r_{i}^{2}}{\mu+r_{i}^{2}}
$$

With a reinjection probability $P\left(r_{i}\right)=c r_{i}$ as displayed by the coupled map system for small values of $r_{i}$, the distribution of laminar phases is given by

$$
P(l)=P\left(r_{i}\right)\left|\frac{d r_{i}}{d l}\right|=\frac{c \mu^{2} e^{-2 \mu l}}{\left(1-e^{-2 \mu l}\right)^{2}} \approx e^{-2 \mu l}
$$

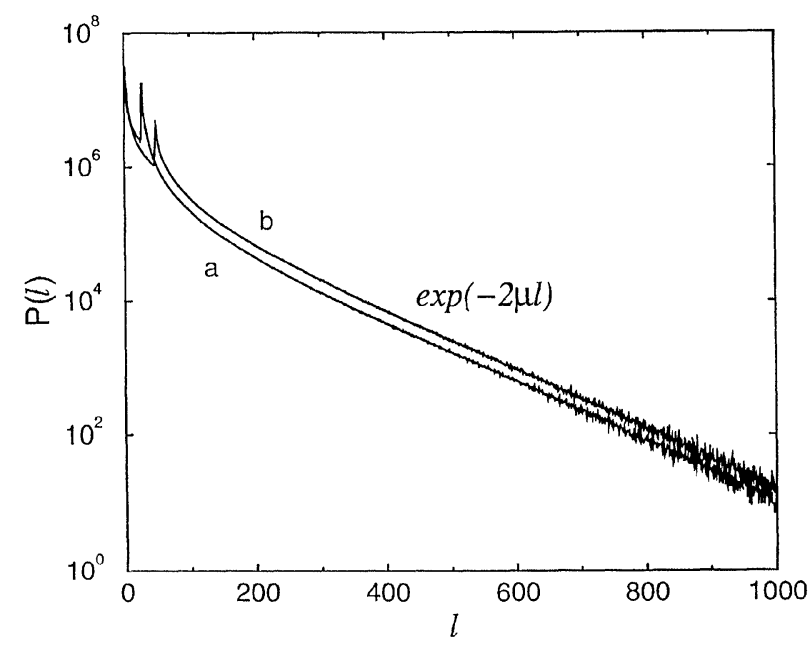

FIGURE 7 For reinjection close to the unstable fixed point, the distribution of laminar lengths follows the theoretically predicted exponential function $P(l) \cong \exp (-2 \mu l)$. The tails of the distributions fluctuate a little because of the relatively few instances of very long laminar phases.

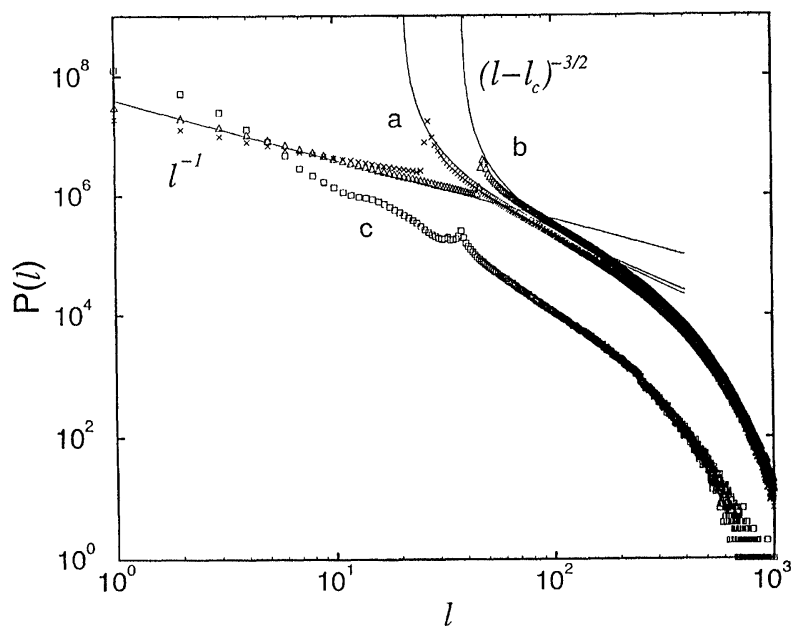

FIGURE 8 Distribution of laminar phases for three different sets of parameter values. Curves (a) and (b) represent the limit of weak coupling $(\varepsilon=0.001)$ whereas curve (c) corresponds to a somewhat stronger coupling $(\varepsilon=0.05)$. For curves (a) and (b) we clearly observe the regions with $l^{-1},\left(l-l_{c}\right)^{-3 / 2}$, and exp $(-2 \mu l)$ behavior. Each curve represents a simulation of more than 100 million laminar phases. 
Here, the last approximation applies for $\mu l \gg 1$. Figure 7 shows numerical results for $P(l)$ obtained with two different sets of parameters. Curve (a) was obtained for $\mu=0.005, b=0.14, \gamma=0.02$ and $\varepsilon=0.001$. Curve (b) applies to $\mu=0.005, b=0.186$, $\gamma=0.13$ and $\varepsilon=0.001$. Both parameter sets have been adjusted so as to secure a uniform reinjection process close to the fixed point.

For distances $r_{i}>f^{2}\left(x_{c}\right)$, the assumption of a uniform reinjection into the unstable manifold no longer applies. Here, $P\left(r_{i}\right) \cong c / r_{i}$, and

$$
P(l) \cong \frac{c \mu}{1-e^{-2 \mu l}} \approx l^{-1}
$$

for $\mu l \ll 1$. This behavior is clearly observed in Figure 8 where the two curves (a) and (b) refer to the same parameter values as above (Fig. 7).

Finally, there is an intermediate region in which the reinjection distance falls below $f^{2}\left(x_{c}\right)$, but close enough to the singularity to render the assumption of uniform reinjection invalid. Here, $P\left(r_{i}\right) \cong$ $r_{i} /\left(r_{i}-f^{2}\left(x_{c}\right)\right)$, and

$$
P(l) \cong\left(l-l_{c}\right)^{-(3 / 2)}
$$

with

$$
l_{c}=-\frac{1}{2 \mu} \ln \frac{\left(f^{2}\left(x_{c}\right)\right)^{2}}{\left(f^{2}\left(x_{c}\right)\right)^{2}+\mu}
$$

being the length of a laminar phase following a reinjection with $r_{i}=f^{2}\left(x_{c}\right)$.

\section{DISCUSSION}

We have developed a simple example to demonstrate the emergence of type-II intermittency in the subcritical Hopf-bifurcation of an antisymmetrically coupled pair of identical one-dimensional maps. By adjusting the parameters a uniform distribution of reinjection points can be obtained over a two-dimensional region of phase space around the unstable fixed point. Hence, the distribution of long laminar phases follows the theoretically predicted exponential form $P(l) \cong$ $\exp (-2 \mu l)$ [17]. This is in contrast to previous investigations $[20-22,26]$ where the reinjection process has been very nonuniform. At the same time, these investigations have dealt with systems of higher specificity, and their value as illustrative examples is correspondingly smaller. Reminiscent of the singularity produced by the critical point, the distribution of short laminar phases shows nongeneric variations. However, these variations also agree well with our theoretical predictions.

According to theory [17], the scaling behavior of the average length of the laminar phases immediately above the subcritical Hopf-bifurcation should follow

$$
\langle l\rangle \approx \ln \frac{1}{\mu-\mu_{0}} \cong \ln \frac{1}{\mu}
$$

with $\mu_{0} \cong-(1 / 2) \varepsilon^{2}$ being the bifurcation point. Figure 9 shows a plot of $\langle l\rangle$ as a function of $\mu$ for three different parameter combinations: $\gamma=0.02$ and $b=0.140$ for curve (a), $\gamma=0.13$ and $b=0.186$ for the intermediate curve, and $\gamma=0.2$ and $b=0.193$ for curve (c). In all cases $\varepsilon=0.001$. The slopes of the curves in the logarithmic plot are all reasonably close to -1 , confirming the prediction (5.1). By varying $\gamma$ and $b$ one can change the range over which the reinjection process is approximately uniform.

It is interesting to note that due to its twodimensional character, the behavior resulting from the subcritical Hopf-bifurcation (type-II intermittency) is hyperchaos with two positive Lyapunov exponents in the limit of low coupling. Figure 10 shows the variation of the two Lyapunov exponents as functions of $\mu$ for $\varepsilon=0.05$. The other parameters are $\gamma=0.02$ and $b=0.14$. These exponents display an abrupt jump at the bifurcation point $\mu_{0} \cong-1 / 2 \varepsilon^{2}$ where 


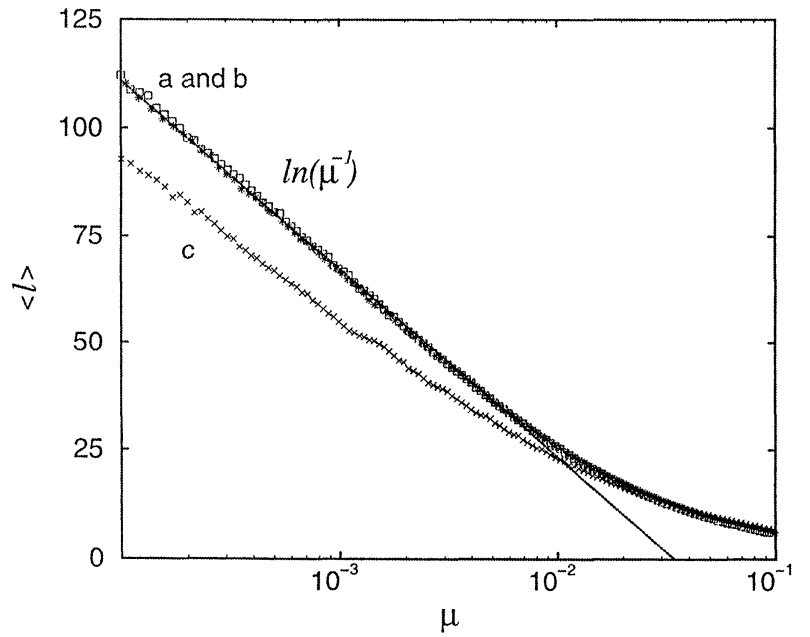

FIGURE 9 Scaling behavior of the average length $\langle l\rangle$ of the laminar phases immediately above the subcritical Hopf-bifurcation for three different sets of parameters. Theory predicts a scaling of the form $\langle l\rangle \cong \ln (1 / \mu)$ as shown by the straight line.

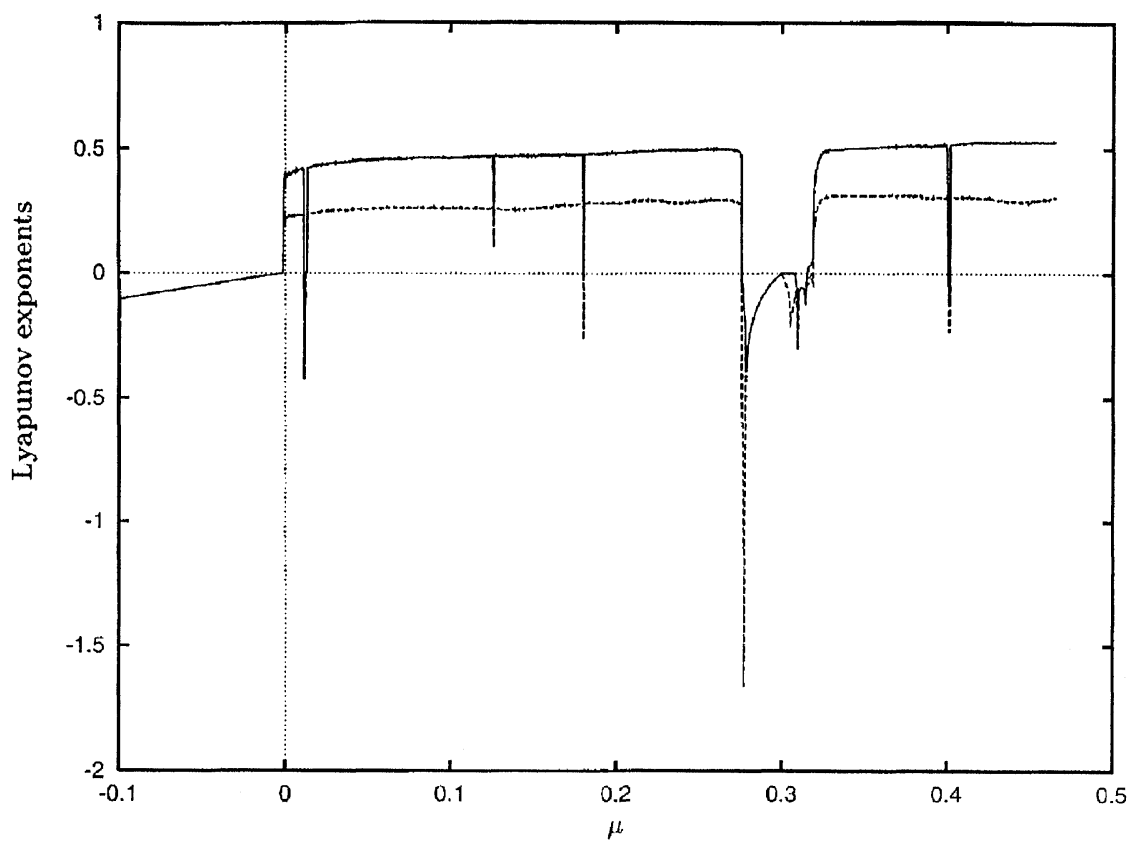

FIGURE 10 Variation of the two Lyapunov exponents as functions of the bifurcation parameter $\mu$. Note how the hyperchaotic behavior arises abruptly in the bifurcation point $\mu_{0} \cong-1 / 2 \varepsilon^{2}$.

type-II intermittency appears. For larger values of $\mu$, intervals exist in which the two maps synchronize to produce a periodic or a quasiperiodic attractor.
Figure 11a shows a brute force bifurcation diagram for the system with the coupling strength $\varepsilon$ as a parameter and Figure $11 \mathrm{~b}$ shows the corresponding variation of the two Lyapunov exponents. 

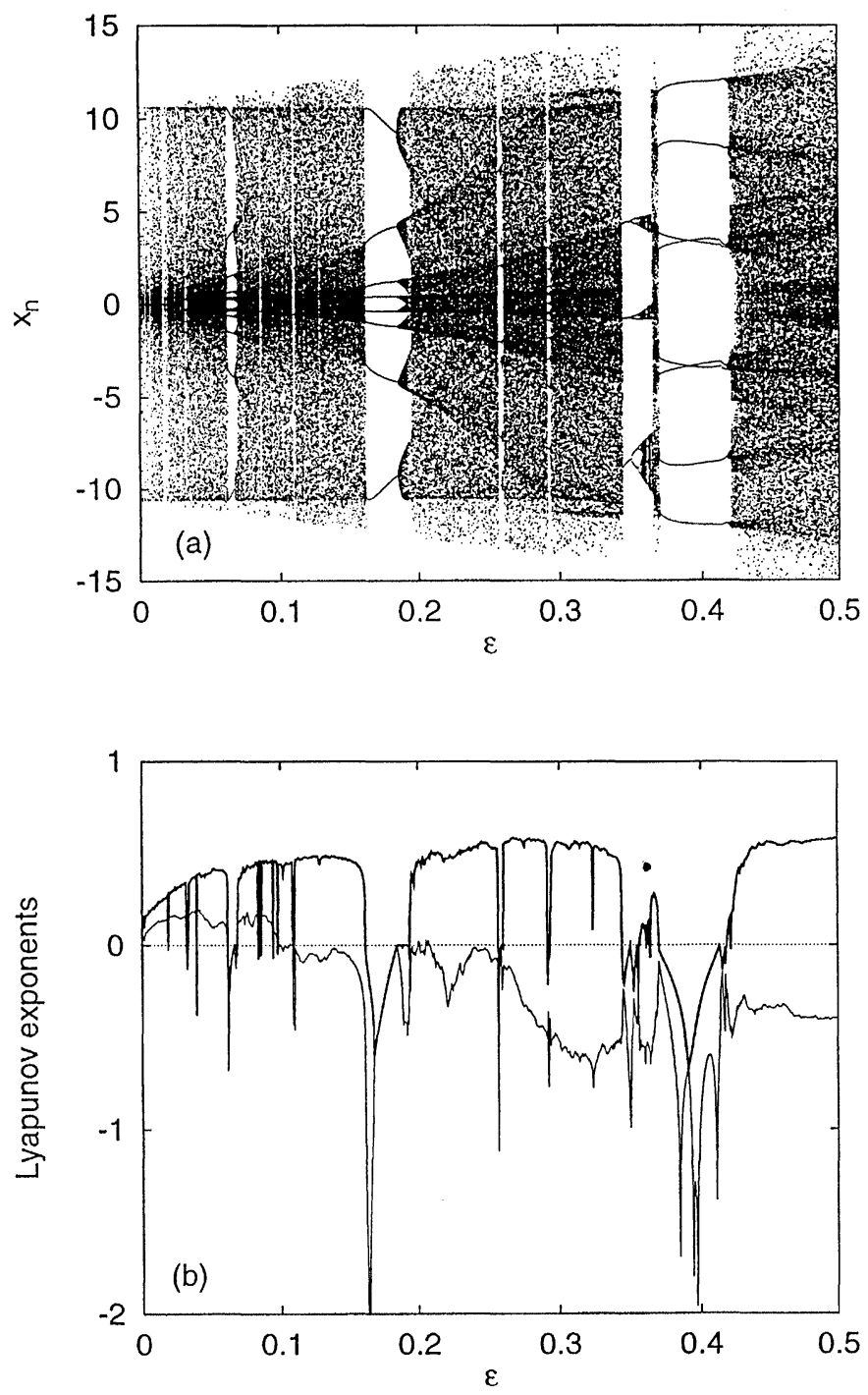

FIGURE 11 Bifurcation diagram (a) and Lyapunov exponents (b) with the coupling strength $\varepsilon$ as a parameter. Note the Hopfbifurcations that occur in several of the periodic windows. Parameters are $\gamma=0.02, b=0.12$, and $\mu=0.001$.

Again, we observe the intervals of synchronization between the maps. Most of these intervals end in a supercritical Hopf-bifurcation followed by a crisis that reestablishes the hyperchaotic behavior. This is typical for two-dimensional systems when the symmetry prevents them from collapsing into a nearly one-dimensional behavior.

To conclude our discussion, finally Figure 12 shows a phase plot of the hyperchaotic attractor existing for $\mu=0.001, \gamma=0.02, b=0.12$, and $\varepsilon=0.10$. The so-called folded-towel structure displayed by this attractor arises through the continuous stretching and folding in two independent directions of phase space. As described by Maistrenko et al. [25] the hyperchaotic attractor is bounded to the so-called absorbing area by the critical curves of the coupled map system. 


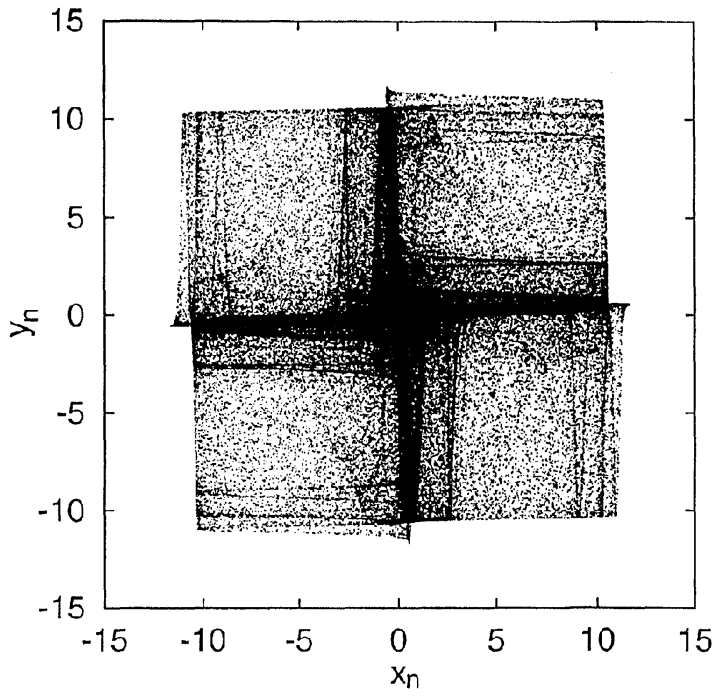

FIGURE 12 Hyperchaotic attractor existing in the coupled map system for $\mu=0.001, \gamma=0.02, b=0.12$, and $\varepsilon=0.10$. The type-II intermittency transition clearly represents a route to hyperchaos.

\section{Acknowledgments}

We would like to express our thanks to $\mathrm{M}$. Andersen and N. Carlsson for contributions to the initial phases of this work. T. Bountis gratefully acknowledges the hospitality of the Chaos Group at The Technical University of Denmark where part of the work was performed under the auspices of the HCM Network CHRXCT93-0331 of the EEC. Support from the Danish Natural Science Foundation is also acknowledged.

\section{References}

[1] Frøyland, J. (1983). Some Symmetric, Two-Dimensional, Dissipative Maps, Physica, D8, 423-434.

[2] Yuan, J.-M., Tung, M., Feng, D. H. and Narducci, L. M. (1983). Instability and Irregular Behavior of Coupled Logistic Equations, Phys. Rev., A28, 1662-1666.

[3] Kuznetsov, S. P. (1985). Universality and Scaling in the Behavior of Coupled Feigenbaum Systems, Radiofizika, 28, $991-1007$.

[4] Hogg, T. and Hubermann, B. A. (1984). Generic Behavior of Coupled Oscillators, Phys. Rev., A29, 275-281.

[5] Van Buskirk, R. and Jeffries, C. (1985). Observation of Chaotic Dynamics of Coupled Nonlinear Oscillators, Phys. Rev., A31, 3332-3357.

[6] Erastova, E. N. and Kuznetsov, S. P. (1991). On the Mechanism for the Onset of Quasiperiodic Oscillations in
Coupled Feigenbaum Systems, Sov. Phys. Tech. Phys., 36, $130-134$.

[7] Reick, C. and Mosekilde, E. (1995). Emergence of Quasiperiodicity in Symmetrically Coupled, Identical Period-Doubling Systems, Phys. Rev., E52, 1418-1435.

[8] Fujisaka, H. and Yamada, T. (1984). Stability Theory of Synchronized Motions in Coupled Oscillator Systems, Prog. Theor. Phys., 69, 32-46.

[9] Pikovsky, A. S. (1984). On the Interaction of Strange Attractors, Z. Phys. B Condensed Matter, 55, 149-155.

[10] Wu, C. W. and Chua, L. O. (1994). A Unified Framework for Synchronization and Control of Dynamical Systems, Int. J. Bifurcation and Chaos, 4, 979-998.

[11] Ott, E., Sommerer, J. C., Alexander, J. C., Kan, I. and Yorke, J. A. (1993). Scaling Behavior of Chaotic Systems with Riddled Basins, Phys. Rev. Lett., 71, $4134-4137$.

[12] Ashwin, P., Buescu, J. and Stewart, J. (1996). From Attractor to Chaotic Saddle: A Tale of Transverse Instability, Nonlinearity, 9, 703-737.

[13] Maistrenko, Yu. L., Maistrenko, V. L., Popovich, A. and Mosekilde, E. (1998). Transverse Instability and Riddled Basins in a System of Two Coupled Logistic Maps, Phys. Rev., E57, 2713-2724.

[14] Platt, N., Spiegel, E. A. and Tresser, C. (1993). On-Off Intermittency: A Mechanism for Bursting, Phys. Rev. Lett., 70, 279-282.

[15] Kendall, B. E., Schaffer, W. M. and Tidd, C. W. (1993). Transient Periodicity in Chaos, Phys. Lett., A177, $13-20$.

[16] Pomeau, Y. and Manneville, P. (1980). Intermittent Transition to Turbulence in Dissipative Dynamical Systems, Comm. Math. Phys., 74, 189-197.

[17] Bergé, P., Pomeau, Y. and Vidal, C. (1984). Order within Chaos: Towards a Deterministic Approach to Turbulence, Wiley and Sons, New York.

[18] Jeffries, C. and Pérez, J. (1982). Observation of a PomeauManneville Intermittent Route to Chaos in a Nonlinear Oscillator, Phys. Rev., A26, 2117-2122.

[19] Yeh, W. J. and Kao, Y. H. (1983). Intermittency in Josephson Junctions, Appl. Phys. Lett., 42, 299-301.

[20] Richetti, P., Argoul, F. and Arneodo, A. (1986). Type-II Intermittency in a Periodically Driven Nonlinear Oscillator, Phys. Rev., A34, 726-729.

[21] Ringuet, E., Rozé, C. and Gouesbet, G. (1993). Experimental Observation of Type-II Intermittency in a Hydrodynamic System, Phys. Rev., E47, 1405-1407.

[22] Herzel, H., Plath, P. and Svensson, P. (1991). Experimental Evidence of Homoclinic Chaos and Type-II Intermittency During the Oxidation of Methanol, Physica, D48, 340 - 352 .

[23] Laugesen, J., Carlsson, N., Mosekilde, E. and Bountis, T. (1997). Anomalous Statistics for Type-III Intermittency in a 1-D Map, Open Systems and Information Dynamics, 4, $393-405$.

[24] Abraham, R. H., Gardini, L. and Mira, C., Chaos in Discrete Dynamical Systems: A Visual Introduction in 2 Dimensions (Springer Verlag, New York, 1997).

[25] Maistrenko, Yu. L., Maistrenko, V. L., Popovich, A. and Mosekilde, E. (1998). Role of the Absorbing Area in Chaotic Synchronization, Phys. Rev. Lett., 80, $1638-1641$.

[26] de Valcarcel, G. J., Roldan, E., Espinosa, V. and Vilaseca, R. (1995). Types I and II Intermittencies in a Cascade Laser Model, Phys. Lett., A206, 359-364. 


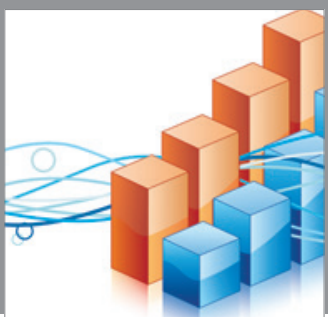

Advances in

Operations Research

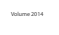

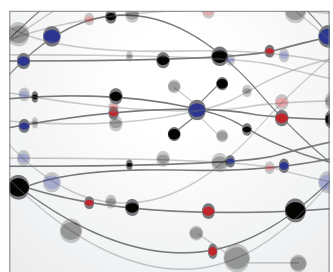

\section{The Scientific} World Journal
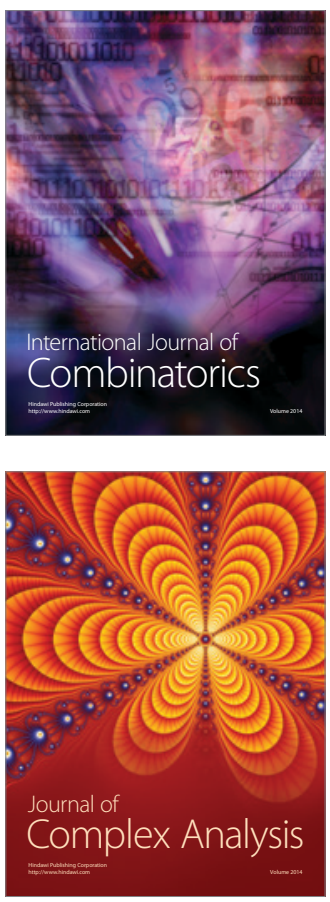

International Journal of

Mathematics and

Mathematical

Sciences
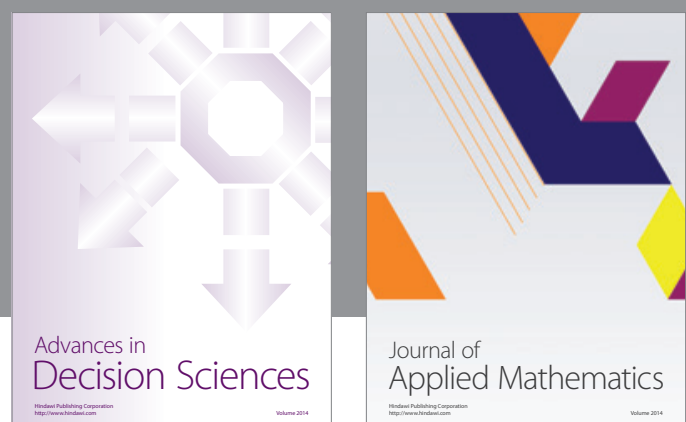

Journal of

Applied Mathematics
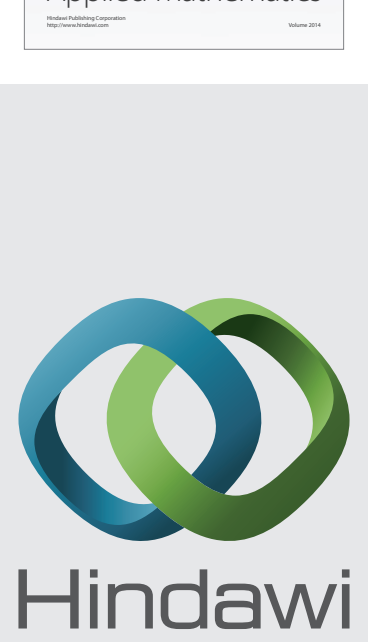

Submit your manuscripts at http://www.hindawi.com
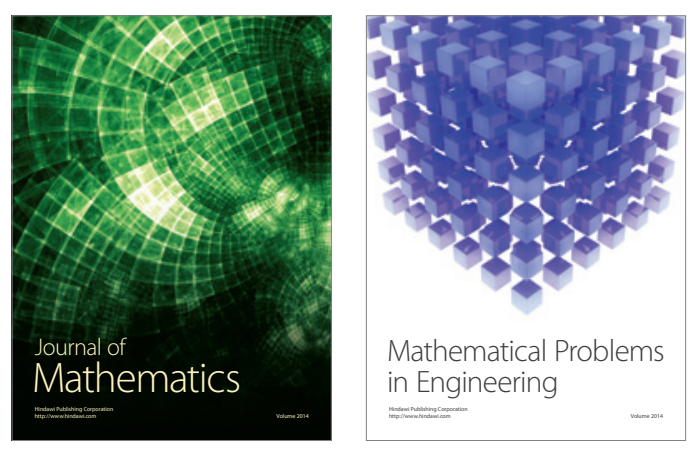

Mathematical Problems in Engineering
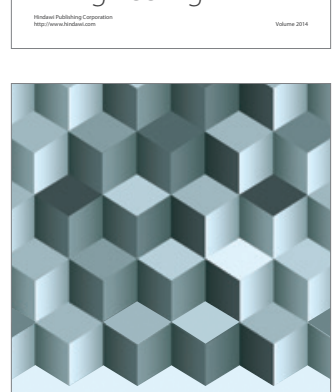

Journal of

Function Spaces
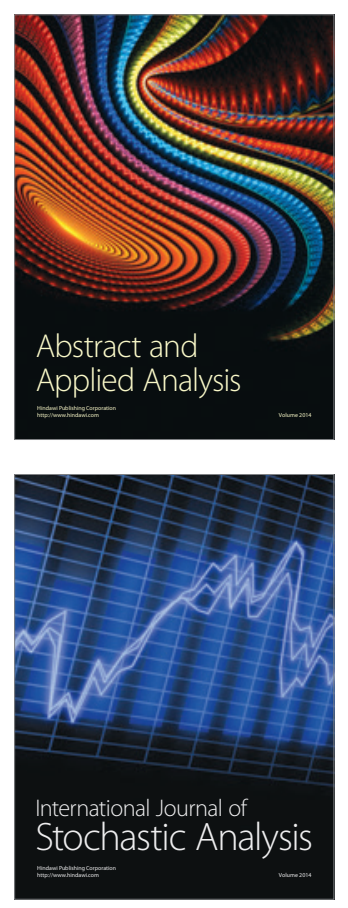

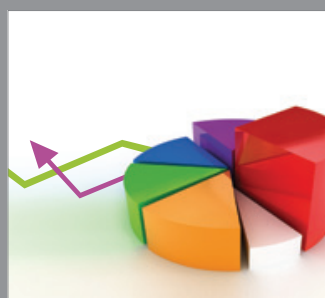

ournal of

Probability and Statistics

Promensencen
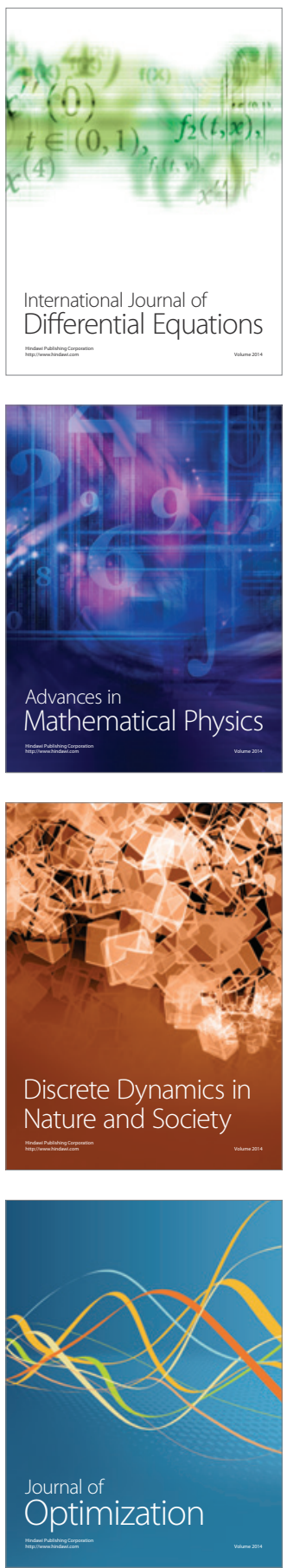